\title{
Volatile elements production rates in a proton-irradiated molten lead-bismuth target
}

\author{
Y. Tall ${ }^{1, \text { a }}$, S. Cormon ${ }^{1}$, M. Fallot ${ }^{1}$, Y. Foucher ${ }^{1}$, A. Guertin ${ }^{1}$, T. Kirchner ${ }^{1}$, L. Zanini ${ }^{2}$, M. Andersson ${ }^{2}$, K. Berg ${ }^{2,3}$, H. Frånberg ${ }^{2,3}$, \\ F. Gröschel ${ }^{2}$, E. Manfrin ${ }^{2}$, W. Wagner ${ }^{2}$, M. Wohlmuther ${ }^{2}$, P. Everaerts ${ }^{3}$, U. Köster ${ }^{3,4}$, H. Ravn ${ }^{3}$, E. Noah Messomo ${ }^{3}$, \\ C. Jost ${ }^{5}$, and Y. Kojima ${ }^{6}$ \\ 1 SUBATECH Laboratory, CNRS/IN2P3-EMN-Université, 44307 Nantes, France \\ 2 Paul Scherrer Institut, 5232 Villigen PSI, Switzerland \\ 3 CERN, 1211 Genève, Switzerland \\ ${ }^{4}$ Institut Laue-Langevin, 38042 Grenoble, France \\ 5 Universtät Mainz, Institut für Kernchemie, Fritz-Strassmann-Weg 2, 55128 Mainz, Germany \\ ${ }^{6}$ Hiroshima University, 1-4-1-Higashi-Hiroshima 7398527, Japan
}

\begin{abstract}
The IS419 experiment at the ISOLDE facility at CERN dedicated to the measurement of production and release rates of volatile elements from an irradiated $\mathrm{Pb} / \mathrm{Bi}$ target by a proton beam of $1 / 1.4 \mathrm{GeV}$ has been completed. The release of $\mathrm{He}, \mathrm{Ne}, \mathrm{Ar}, \mathrm{Br}, \mathrm{Kr}, \mathrm{Cd}, \mathrm{I}, \mathrm{Xe}, \mathrm{Hg}$, Po and At isotopes was investigated at different target temperatures, ranging from $250^{\circ} \mathrm{C}$ to $600^{\circ} \mathrm{C}$. Three experimental methods were used for the mass-separated, ionized beams: i) implantation of short- and medium-lived isotopes in a tape and on-line detection with a HPGe $\gamma$ detector; ii) implantation of longer-lived isotopes in Al foils and off-line detection with a HPGe detector; iii) a Faraday Cup used mainly for stable nuclides. The results were compared with predictions from the FLUKA and MCNPX codes using different options for the intra-nuclear cascades and evaporation/fission models. Results show good agreement with calculations for $\mathrm{Hg}$ and for noble gases. For other elements such as iodine it is apparent that only a fraction of the produced isotopes is released. The results from FLUKA and MCNPX with the INCL4/ABLA models are in general more satisfactory than those obtained using MCNPX with the standard Bertini/Dresner model combination. Interestingly also significant yields of ${ }^{204-210}$ At isotopes were observed. At isotopes are produced either by $\left(\mathrm{p}, \pi^{-} x \mathrm{n}\right)$ charge exchange reactions on ${ }^{209} \mathrm{Bi}$ or by secondary reactions involving ${ }^{3} \mathrm{He}$ and ${ }^{4} \mathrm{He}$. Despite the non-release of polonium from $\mathrm{Pb} / \mathrm{Bi}$ targets at typical operation temperatures, a smaller amount of highly radiotoxic Po isotopes can actually be liberated indirectly as decay daughters of the released astatine.
\end{abstract}

\section{Introduction}

In the development of key experiments in the frame of the research on molten metal targets for Accelerator Driven System (ADS) [1] for the nuclear waste transmutation, the isotope production following the interaction of an intense proton beam with a liquid lead bismuth target is an important issue, in primis for safety reasons. The key experiment for the development of the target in the European roadmap for developing Accelerator Driven Systems for nuclear waste incineration is MEGAPIE [2], which was irradiated in 2006. The aim of MEGAPIE is to demonstrate the feasibility of a liquid lead bismuth eutectic (LBE) target for spallation facilities at a beam power level of about $1 \mathrm{MW}$. During the design phase of such an innovative system, many safety aspects have to be considered. One of them concerns the production of volatile elements during operation. Spallation reactions, in addition to the neutrons, generate radiotoxic waste products. Part of the nuclides produced are more or less volatile at the target operation temperature $\left(300-400^{\circ} \mathrm{C}\right)$. The volatile products diffuse out of the LBE and are collected in a volume on top of the target. It is important to know the total production of volatile products, specially for the noble gases $\mathrm{He}, \mathrm{Ne}$, Ar, $\mathrm{Kr}$ and $\mathrm{Xe}$, the volatile metal $\mathrm{Hg}$ and the halogens $\mathrm{Br}$ and $\mathrm{I}$.

${ }^{a}$ Presenting author, e-mail: yoro.tall@subatech.in2p3.fr
The study of the production and release rates of these volatile elements is important for several reasons: i) some stable gases, and in particular ${ }^{4} \mathrm{He}$ and ${ }^{1-3} \mathrm{H}$, are expected to be produced in relatively large quantities (in the case of MEGAPIE, about 1 liter NTP per month) and a system must be designed to handle safely the gases and avoid excessive pressure buildups. Moreover, it is important to know the production of these light elements to estimate possible damage to the structural materials. ii) The production of radioactive elements is of concern for safety reasons. The long-lived elements are of major concern, but short-lived elements are also of interest for accident scenarios. iii) Another point of interest for studying the release is related to new ISOL (isotope separation online) targets under development e.g. for EURISOL, where also liquid targets are considered for direct isotope production.

In order to study the production and release rates of volatile elements from an irradiated LBE target, an experiment with a molten $\mathrm{Pb} / \mathrm{Bi}$ target was performed at the ISOLDE facility [3] at CERN with proton beams of 1 and $1.4 \mathrm{GeV}$ respectively. Data were also taken with a liquid lead target. Beyond the estimation of the production and release rates of volatile elements in an irradiated LBE target, this experiment will permit to validate the Monte Carlo codes (MCNPX [4] and FLUKA [5]). The obtained results are compared to MCNPX and FLUKA simulations coupled respectively to the evolution codes CINDER90 [6] and ORIHET3 [7]. 


\section{Overview of the experiment}

The experiment was performed at the ISOLDE facility in three runs between 2004 and 2005. The experiment is described in ref. [8]. For the first and the last run, the target consisted of lead-bismuth contained in a tantalum cylinder of $20 \mathrm{~cm}$ length and $1 \mathrm{~cm}$ radius, $75 \%$ filled; the target was irradiated with protons of $1.4 \mathrm{GeV}$ and $1.0 / 1.4 \mathrm{GeV}$ respectively for the first and the third run. The mass of the LBE was $547 \mathrm{~g}$; in order to obtain good release efficiency, for most of the measurements the target temperature was fixed at around $600{ }^{\circ} \mathrm{C}$. In the second run, a liquid lead target was used with a proton beam of $1.4 \mathrm{GeV}$ and a target temperature fixed at $520^{\circ} \mathrm{C}$. These temperatures are higher than the range of the LBE temperatures in MEGAPIE during operation, which varies from $300{ }^{\circ} \mathrm{C}$ to $400^{\circ} \mathrm{C}$ depending on the position inside the target. Having performed the experiment at higher temperatures than MEGAPIE will allow, to conclude, in case the release of a specific isotope is not observed at $600{ }^{\circ} \mathrm{C}$, no release is expected in MEGAPIE for the same isotope at $300-400^{\circ} \mathrm{C}$, even for much longer irradiation times.

Various measurement techniques are available at ISOLDE and can be subdivided in two categories according to the halflife of the isotopes.

- Off-line measurements using $\gamma$-spectroscopy are used for isotopes with half-lives from about 5 minutes to few weeks.

- On-line measurements (two procedures can be used to collect data): the Faraday Cup and the tape station measurement. The Faraday Cup is most useful for measuring stable isotopes or long-lived radioisotopes. The tape station measures the production rate of radioisotopes with a very short half-life. After collection of the radioactive ion beams on the tape, it is transported to a germanium detector which carries out the on-line measurements.

We investigated the release of $\mathrm{He}, \mathrm{Ne}, \mathrm{Ar}, \mathrm{Br}, \mathrm{Kr}, \mathrm{Cd}, \mathrm{I}, \mathrm{Xe}$, $\mathrm{Hg}$, Po and At radioisotopes. During the measurements, most attention was concentrated on those isotopes that are critical for the operation of an ADS target such as MEGAPIE. A selection of the data obtained is presented in this paper, with emphasis on the $\gamma$-spectroscopy data.

The release of the volatile elements from an ISOL target is a complex process $[9,10]$. The volatile species formed after the spallation reaction diffuse first in the liquid. The diffusion speed in the bulk material depends on the target material, the temperature and the diffusing species. The elements effuse through the target container, the transfer line and the ion source. They are ionized by a plasma ion source, then accelerated to $60 \mathrm{keV}$ and sent to the magnetic mass separators and to the beam lines where the measuring stations are placed. Part of the produced elements is lost during diffusion, effusion, ionization and transmission due to their possible chemical reactivity, sticking to the wall, condensation, leaks and or passing through the ion source without being ionized. In order to obtain the in-target production rates of the volatile elements, one must take into account the different efficiencies (of the ion source, the transmission line, the mass separator and the detector). Temperature dependence of the yields were measured for several isotopes (fig. 1 for a target temperature

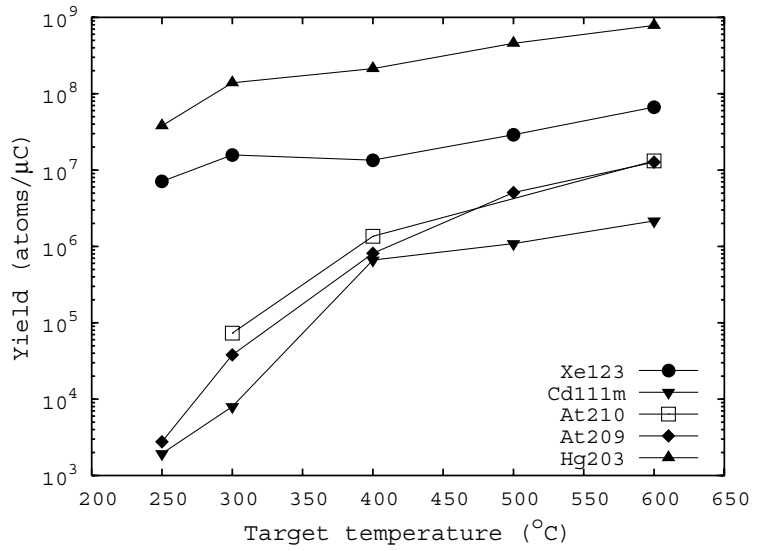

Fig. 1. Temperature dependence of the measured yields (atoms $/ \mu \mathrm{C}$ ) for the indicated isotopes.

from $250{ }^{\circ} \mathrm{C}$ to $600{ }^{\circ} \mathrm{C}$ ). While it is expected that for $\mathrm{Cd}, \mathrm{Hg}$ and At isotopes the release increases significantly with the temperature, it is somewhat surprising that for $\mathrm{Xe}$ isotopes the release still increases from $500{ }^{\circ} \mathrm{C}$ to $600{ }^{\circ} \mathrm{C}$. Further investigations showed that the release time of noble gases in the LBE target is a long process (of the order of 10-30 minutes), even at high temperature, thus indicating that probably a constant release was not reached for the noble gases when the temperature measurements were taken. Nevertheless, only a post irradiation analysis, by mass spectroscopy, of samples taken from the target will give an answer, whether the release at $600{ }^{\circ} \mathrm{C}$ was complete or not. Such a measurement will be performed in 2007 .

\section{Results}

The data obtained with off-line and on-line $\gamma$-spectroscopy were compared to FLUKA and MCNPX calculations. The production rates from the two Monte Carlo codes were coupled with the evolution codes ORIHET3 and CINDER90 respectively. In the case of MCNPX, results are shown here with two different model combinations for the intranuclear cascade and evaporation/fission models: the default Bertini/Dresner combination, and the newer INCL4/ABLA model combination.

Noble gases. Figure 2 shows the production rates of xenon isotopes. We obtain good agreement between data and simulations in the case of FLUKA and MCNPX when combined with the INCL4 intra-nuclear cascade model and the ABLA evaporation/fission option. The Bertini intra-nuclear cascade model with the Dresner evaporation/fission option when implemented into MCNPX overestimates the production rates of xenon isotopes following the interaction of a proton beam of $1.4 \mathrm{GeV}$ in a $\mathrm{Pb} / \mathrm{Bi}$ target. While for a proton beam of 1.0 GeV, MCNPX (Bertini/Dresner or INCL4/ABLA) and FLUKA reproduce well the data for the Krypton isotope production rates (see fig. 3). The same is observed for xenon isotope production rates at the same proton beam energy.

Krypton isotopes, like $\mathrm{Xe}$ isotopes, are produced in the later stage of the spallation process, the fission of highly 


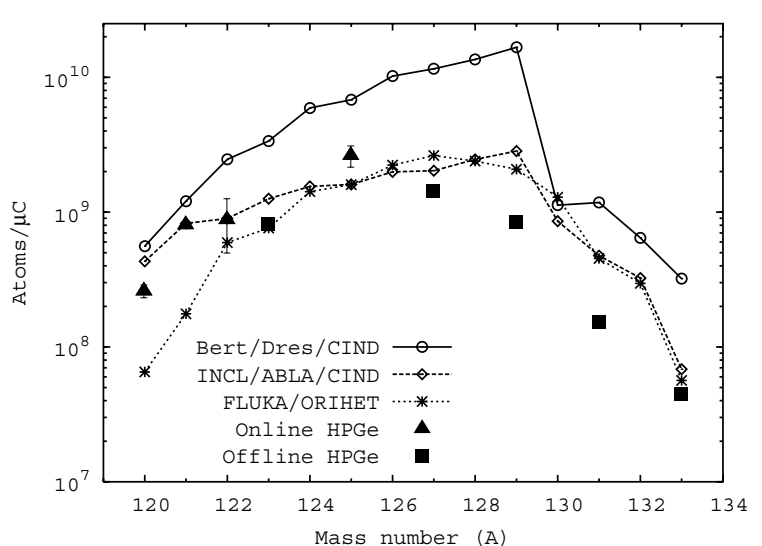

Fig. 2. Production rates for $\mathrm{Xe}$ isotopes for $1.4 \mathrm{GeV}$ protons at $600^{\circ} \mathrm{C}$ target temperature. Measured points with on-line and offline HPGe detector (respectively black filled triangles and squares) compared with calculations: open circles: MCNPX (Bertini/Dresner model combination); open diamonds: MCNPX (INCL4/ABLA); stars: FLUKA.

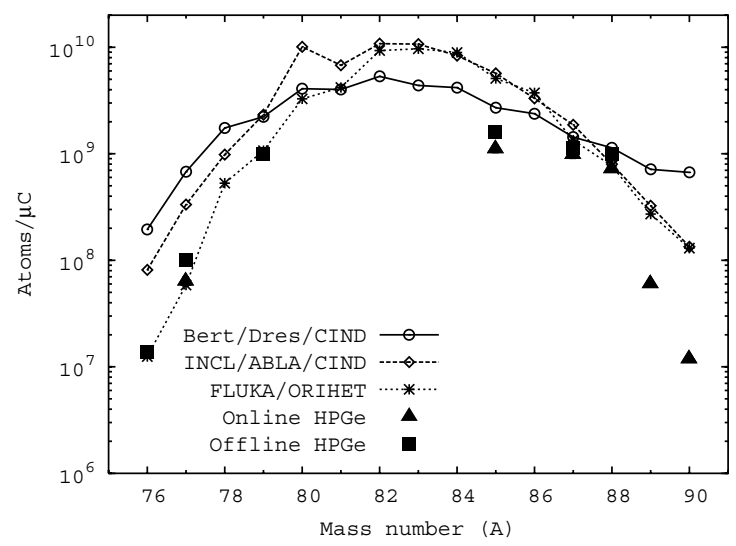

Fig. 3. Same as figure 2 but for $\mathrm{Kr}$ isotopes production rates, for $1 \mathrm{GeV}$ protons.

excited spallation fragments, or as a two-step process due to neutron induced fission from high-energy spallation neutrons. Evaporation/fission models are therefore responsible for the differences between the codes observed in figure 2. However, in this case we cannot conclude which model is better, even though the isotope mass distributions seem to indicate that MCNPX with INCL4/ABLA and FLUKA give the best results.

Mercury. In figure 4 the measured cumulative production rates at $1.4 \mathrm{GeV}$ for radioactive $\mathrm{Hg}$ isotopes are presented. In general the agreement between the data is good. Also, the simulations reproduce well the production rates given by the measurements. Longer-lived $\mathrm{Hg}$ isotopes are expected to be completely released at the temperature of $600^{\circ} \mathrm{C}$. We note a trend, that is observed in all the mass distribution plots, that for isotopes in the low and high tails of the distributions the data are lower than the calculations. Due to the long release time of volatile elements in a liquid target, part of the shorterlived isotopes decay inside the target prior to release.

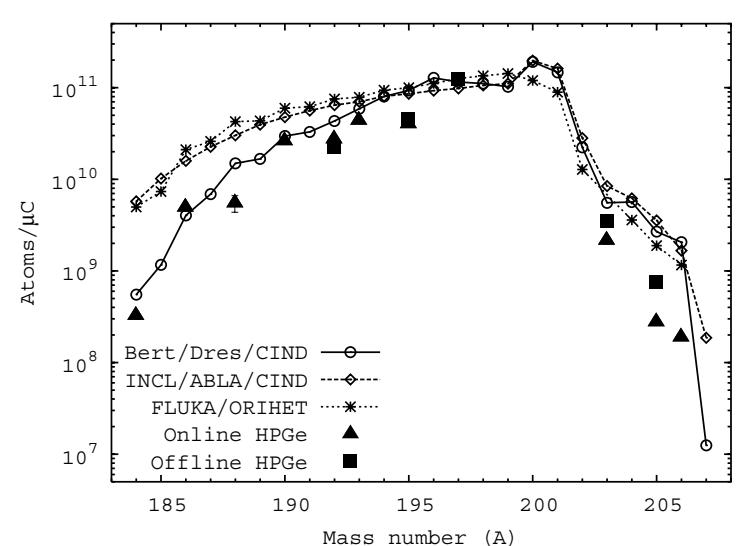

Fig. 4. Same as figure 2 but for $\mathrm{Hg}$ isotopes production rates, for $1.4 \mathrm{GeV}$ protons.

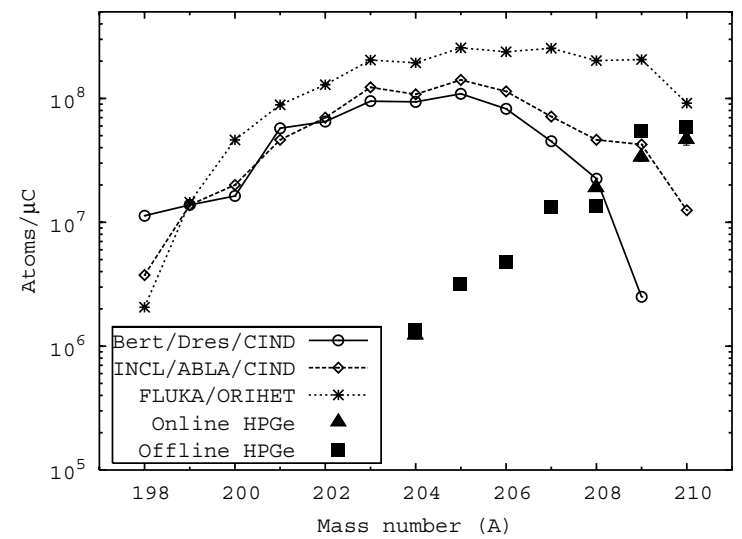

Fig. 5. Release rates of At isotopes at $600^{\circ} \mathrm{C}$, for $1.4 \mathrm{GeV}$ protons.

Astatine and polonium. The astatine isotopes have short halflives and are produced at relatively low rates in the target. Astatine release is therefore not a safety issue. However, astatine $\beta$ decays to polonium which has highly radiotoxic isotopes since they are alpha emitters and some of them are very long-lived, e.g., 2.9 and 102 years for ${ }^{208} \mathrm{Po}$ and ${ }^{209} \mathrm{Po}$ respectively. It is therefore important to measure the release rates of astatine isotopes. Figure 5 shows the release rates of astatine isotopes when a proton beam of $1.4 \mathrm{GeV}$ energy interacts with the $\mathrm{Pb} / \mathrm{Bi}$ target. The results of the measurements agree with each other while the simulations give results higher than the measurements. The release rates of astatine have similar values for proton beam energies of $1.0 \mathrm{GeV}$ and $1.4 \mathrm{GeV}$. The discrepancy between the calculated and measured values is probably due to incomplete modeling in the Monte Carlo codes, especially in the transport of light particles such as alpha, ${ }^{3} \mathrm{He}$ and pions. These light particles play a major role in the production of astatine isotopes [11]. The dominating direct reactions are ${ }^{209} \operatorname{Bi}\left(\mathrm{p}, \pi^{-} x \mathrm{n}\right)^{210-x}$ At. However, for a thick target as used in this experiment, secondary reactions must be taken into account:

- ${ }^{209} \mathrm{Bi}\left({ }^{3} \mathrm{He}, x \mathrm{n}\right)^{212-x} \mathrm{At}$ induced by spallation-produced ${ }^{3} \mathrm{He}$, - ${ }^{209} \mathrm{Bi}\left({ }^{4} \mathrm{He}, x \mathrm{n}\right){ }^{213-x} \mathrm{At}$ induced by spallation-produced ${ }^{4} \mathrm{He}$.

Even though such reactions are included in the FLUKA and MCNPX transport, the experimental trend is not reproduced, 


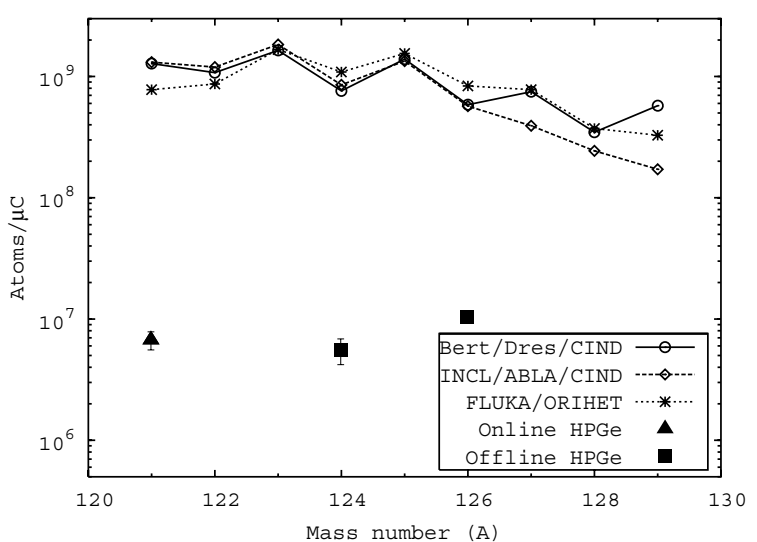

Fig. 6. Release rates of iodine isotopes at $600{ }^{\circ} \mathrm{C}$, for $1 \mathrm{GeV}$ protons.

indicating that model refinements are needed. No direct release of polonium isotopes was observed, only from astatine decay.

Other isotopes. Other less volatile elements were studied. Some release of $\mathrm{Br}$ and $\mathrm{Cd}$ isotopes was detected, with a maximum release (compared to calculations) of about $0.3 \%$ at $600{ }^{\circ} \mathrm{C}$. Release of iodine isotopes is shown in figure 6 and compared with calculations. The production rates measured are more than ten times lower than the predictions of the Monte Carlo simulations. This is in agreement with the study in refs. $[12,13]$ on the release rates of volatile elements from lead bismuth targets which indicates that the release rate of iodine is around $1 \%$ at $600{ }^{\circ} \mathrm{C}$.

\section{Discussion}

The complex process of production, diffusion and release of volatile elements in a molten metal target has been investigated at CERN/ISOLDE, giving very important results of direct application for experiments such as MEGAPIE. Despite the complexity of the processes involved, the data show good agreements with calculations. The capability of Monte Carlo codes used in the MEGAPIE neutronic design phase, in predicting isotope production, was thus confirmed. It must be noted that disagreements are found especially in the tails of the mass distributions, since shorter lived nuclides partially decay in the target before being released. Due to the complex release path, we were not able to correct for partial release (as it is done in the case of solid targets). But the obtained data allow us to conclude that in most of the cases for residues from the spallation-fission reactions FLUKA and MCNPX(INCL4/ABLA) are more reliable than the Bertini/Dresner model, one good example being the $\mathrm{Xe}$ release at $1.4 \mathrm{GeV}$ proton energy.

The release of longer-lived $\mathrm{Hg}$ and noble gases isotopes is complete or nearly complete at $600{ }^{\circ} \mathrm{C}$. However, given the observed temperature dependence in the release, a post-irradiation analysis will be performed in PSI (Villigen, Switzerland) in the course of 2007. Mass spectrometry measurements will indicate if traces of such isotopes are still present in the target.

For other isotopes, such as $\mathrm{Cd}, \mathrm{Br}, \mathrm{I}$, and At the release is only partial, as expected. A very interesting case is At, the release of which was observed for the first time from a molten ISOL target. The measured astatine release rates spotted some deficiencies in the present Monte Carlo models.

Supported by the EU projects TARGISOL (contract HPRI-CT-200150033), MEGAPIE-TEST (contract FIKW-CT-2001-00159) and EUROTRANS (contract FI6W-CT-2004-516520).

\section{References}

1. The European Technical Working Group on ADS, A European Roadmap for Developping Accelerator Driven System (ADS) for Nuclear Waste Incineration, ENEA, Roma, 2001.

2. G.S. Bauer et al., J. Nucl. Mat. 296, 17 (2001).

3. E. Kugler, Hyperfine Interactions 129, 23 (2000).

4. L.S. Waters et al., MCNPX User's Manual Version 2.4.0, LA-CP-02-408 (2002).

5. A. Fassò et al., in Proceedings of the Monte Carlo 2000 Conference Lisbon, edited by A. Kling et al., F. Barao, M. Nakagawa, L. Tavora, P. Vaz (Springer-Verlag, Berlin, 2001), p. 159.

6. W.B. Wilson, T.R. England, P. Möller, A Manual for CINDER'90 Version 06.1 Codes and Data, LA-UR-06-draft, Los Alamos National Laboratory, New Mexico (2006).

7. F. Atchison, H. Schaal, Orihet 3 - Version 1.12, A guide for users, March 2001.

8. L. Zanini et al., Volatile Elements Production Rates in a 1.4-GeV Proton-Irradiated molten Lead-Bismuth Target, in International Conference on Nuclear Data for Science and Technology, edited by R.C. Haight et al. (Melville, New York, 2005), p. 1525.

9. U. Köster, Ausbeuten und Spektroskopie radioaktiver Isotope bei LOHENGRIN und ISOLDE, Ph.D. thesis, Technische Universität München, 2000, and references therein.

10. U. Köster for the ISOLDE Collaboration, Radiochim. Acta 89, 749 (2001).

11. J.L. Clark et al., Inclusive $\pi^{-}$production with $200 \mathrm{MeV}$ protons: Radiochemical study of the ${ }^{209} B i\left(p, \pi^{-} x n\right)^{210-x}$ At reactions, Phys. Rev. C 26, 2073 (1982).

12. J. Neuhausen et al., Radiochim. Acta 92, 917 (2004).

13. J. Neuhausen et al., Radiochim. Acta 93, 155 (2005). 